\title{
Interpretation of the Computerized Tomographic Scan
}

\author{
M. BANNA
}

SUMMARY: This paper is based on the experience gained from over three thousand examinations by computerized tomography (CT). The appearances of various intracranial lesions are presented. The reliability and limitations of this new technique are also described and some pitfalls in scan interpretation are mentioned.

RÉSUMÉ: Le présent article décrit l'aspect de différentes lésions intracrâniennes au CT-Scan. Il décrit aussi la fiabilité et les limites de cette nouvelle technique et cite quelques pièges dans linterprétation du scan, ceci basé sur lexpérience acquise' avec plus de 3000 examens.

\footnotetext{
From the Departments of Radiology of the Hamilton General Hospital and McMaster University
} Medical Centre, Hamilton. Ontario.

Reprint requests to: Dr. M. Banna, Department of Radiology, McMaster University Medical Centre, 1200 Main Street West, Hamilton. Ontario Canada L8S 4 J9.

\section{INTRODUCTION}

The principles of computerized tomography have been outlined in a previous publication (Banna and Porteous, 1976). The present paper describes our experience with the scan in various intracranial lesions. Our findings correlate well with others (Davis and Pressman, 1974; Paxton and Ambrose, 1974). In this paper a tissue is described as either of high or low density relative to the surrounding brain. The normal appearances are shown in Figure 1.

Intracranial lesions may be broadly classified into two categories.

A. High density lesions: These include any type of intracranial calcification, hemorrhage, giant aneurysm, benign tumors such as meningioma, neurofibroma, pituitary adenoma and a minority of malignant intracranial neoplasms.

B. Low density lesions: These include all fat containing tumors such as lipoma, cholesteatoma and craniopharyngioma, cystic lesions within or outside the brain, and the majority of malignant neoplasms and cerebral infarctions or edema. Each of these will be separately considered.

\section{INTRACRANIAL CALCIFICATION}

All types of intracranial calcification are easily detectable on the $\mathrm{CT}$ scan even if the density is not sufficient to cast a shadow on the conventional skull radiograph (Figure 2 ). The incidence of pineal and choroid calcification is higher on the scan than on conventional radiography and we have observed calcification in the basal ganglia in a number of patients in whom no clinical, biochemical or other radiological abnormality could be shown (Figure 3).

\section{INTRACRANIAL HEMATOMA}

In intracerebral hemorrhage the scan is probably the quickest and most reliable means of investigation. It is superior to angiography which may be normal or show vascular displacement without specific information about the underlying pathology. Intracerebral hematoma appears as an homogeneous, high density area which may be globular or elliptical in shape and may be surrounded by a smaller darker area of edematous or necrotic brain (Figure 4). In most cases the appearances are pathognomonic. Sometimes the hematoma may be mistaken for an intracranial neoplasm. This may occur if the lesion is surrounded by a larger area of brain edema that is enhanced by contrast (Figure 5).

Hemorrhage into the ventricular system is usually an ominous event but a few patients survive. In them, serial CT scans show absorption of the hematoma (Figure 6).

The shape of acute subdural and epidural hematomas is similar by scan and angiographic examination. The former are semilunar and the latter are usually biconvex (Figure 7). We have found that a small subdural hematoma, particularly in relation to the skull base, may be easily overlooked on the CT scan. We have also seen an acute subdural hematoma which was well demarcated on the CT scan but the angiogram revealed that it was precipitated by a bleeding aneurysm in the contralateral hemisphere. Upon bursting of the aneurysm the patient lost consciousness, fell and developed a subdural hematoma. Bizarre as it may be, this diagnosis could not have been made on the scan alone.

Giant berry aneurysms are globular in shape. In contrast to angiographic studies, it is the clotted part of the aneurysm which is most dense 
on the preliminary CT scan. Nonclotted blood within an aneurysm or a massive vascular malformation may only be apparent following contrast enhancement (Figure 8). Small lesions, either aneurysms or vascular malformations may not be detectable on the CT scan.

\section{BENIGN INTRACRANIAL TUMORS}

The majority of intracranial meningiomas appear moderately dense on the preliminary scan examination and their density is often well enhanced following the intravenous injection of contrast medium. The lesion is usually well defined but may be surrounded by brain edema. Hyperostosis if excessive, may be apparent on the scan. If these features are considered in the light of the known sites of meningiomas, a histological diagnosis can often be made (Figures 9 and 10). The pitfalls we have encountered should be mentioned. Massive frontal exostosis masked a small meningioma approximately one inch in diameter. In another case the tumor contained enough lipomatous material thus reducing its density and it could not be seen. From approximately twenty meningiomas, the tumor was thought to be a glioma on two occasions. (Figure 11).

In cases of acoustic neuroma (Figure 12) and pituitary adenoma (Figure 13) it is the extraosseous part of the tumor which is apparent on the CT scan. Lesions confined to the sella or within the auditory canal are masked by the bone density and cannot be seen.

\section{MALIGNANT INTRACRANIAL NEOPLASMS}

Primary and secondary malignant brain tumors have a wide spectrum of densities which may be slightly higher or lower than the density of normal brain. This is not surprising in view of the enormous variability of these tumors. Because of their invasive nature they often appear irregular in outline and are surrounded by varying degrees of brain infiltration and edema. The majority are enhanced by contrast but usually less than meningiomas. They are seldom as homogeneous or as dense as the meningiomas (Figure 14). Following contrast enhancement, the darker necrotic part of the tumor and surrounding infiltration are more clearly identified. Cerebral metastases have the same features as gliomas but can be recognized with certainty if multiple (Figure 15).

\section{FAT CONTAINING TUMORS}

In this category the tumor which is most distinctive is the lipoma of the corpus callosum. It is characterized by being very low in density and mid-line in position (Figure 16). The density of an intracranial cholesteatoma (dermoid or epidermoid cyst) is often not as low as the lipoma (Figure 17) and neither lesion is enhanced by contrast.

The consistency of craniopharyngiomas varies from an entirely cystic to a stony hard tumor and the density varies similarly. Cystic lesions often show low absorption values and the cyst wall may be enhanced by contrast (Figure 18). The solid non-calcified type of craniopharyngioma is difficult to differentiate from other invasive lesions such as an optic chiasm glioma (Figure 19).

\section{CYSTIC NON-LIPID CONTAINING LESIONS}

These may be extracerebral such as chronic subdural hematomas and arachnoid cysts. They may also be intracerebral, i.e. cystic glioma, hemangioblastoma and colloid cysts. The cystic component of these lesions has a density similar to cerebrospinal fluid and their location within or outside the brain can be easily recognized on the scan. The majority of chronic subdural hematomas are biconvex in shape, but contrary to what is generally found with angiography some may be crescentic and of the same shape as the acute variety (Figure 20).

Arachnoid cysts are closely related to chronic subdural hematomas. In some cases they are probably the same type of lesion. Some are developmental and a few are post-inflammatory. They may be found over the cerebral hemispheres, beneath the temporal lobes, in the posterior fossa, in the hypophyseal area or in relation to the quadrigeminal plate. They have the same density as cerebrospinal fluid, their margins are well defined, they are not surrounded by brain edema and are not enhanced by contrast.

Cystic gliomas and hemangioblastomas contain a solid portion which unlike arachnoid cysts and cholesteatomas may be enhanced by contrast. However, we have several examples where the solid component of an hemangioblastoma was shown by angiography but was difficult to detect on the CT scan.

In colloid cysts of the third ventricle, hydrocephalus is limited to the lateral ventricles, the fourth being normal in size. The cyst itself is not invariably apparent on the scan but in some it may be enhanced by contrast (Baker et al., 1974; Sage et al., 1975).

\section{CEREBRAL INFARCTION}

Lesions due to cerebral infarctions may be massive or focal, superficial or deep, single or multiple. They all have a low density approaching that of cerebrospinal fluid (Figure 21). Rarely, they are slightly enhanced by contrast. Ventricular displacement is often comparatively slight in relation to the size of the lesion but massive shifts may be seen.

Superficial infarction is usually semilunar or wedge shaped, the cortex being more affected than the white matter. This feature often helps to differentiate between infarction and infiltrating glioma. In a mass lesion if one can identify a rim of normal brain cortex, infarction can be excluded (Figure 22). Old infarction may be associated with localized or generalized dilatation of the ipsilateral ventricle (Figure 22C).

Areas of deep focal infarction are often difficult to differentiate from early tumor infiltration but the clinical history may be helpful and serial scan examinations may be indispensible.

With cerebral infarction it is perhaps appropriate to include brain abscess. In the early stages of abscess formation, there is an irregular non-homogeneous low density area 
representing necrotic and edematous brain. As the lesion becomes more chronic, an abscess wall can be identified following contrast enhancement (Figure 23). In the past, positive contrast media was often instilled in the abscess cavity to help assess its size and response to treatment. With the availability of the CT scan, this undesirable introduction of foreign material is no longer necessary.

\section{ESTIMATION OF VENTRICULAR SIZE}

The CT scan has eliminated the need for pneumoencephalography and ventriculography in the investigation of many neurological disorders such as brain atrophy, pseudotumor cerebri, hydrocephalus secondary to trauma, meningeal infection, subarachnoid hemorrhage, Arnold-Chiari malformation and many other diseases (Figures 24 and 25 ). The CT scan is also a reliable method for testing the patency of any ventricular shunting device. Not withstanding these advantages, there are still a number of cases in which ventriculography is indicated.
In this respect we wish to draw attention to a major drawback of oily contrast media. The presence of such material within the cranium restricts the interpretation of the scan, a point which should be remembered during myelography. Spilling of the contrast media into the basal cisterns deprives the patient from the usefulness of computerized tomography if the need for it ever arises.
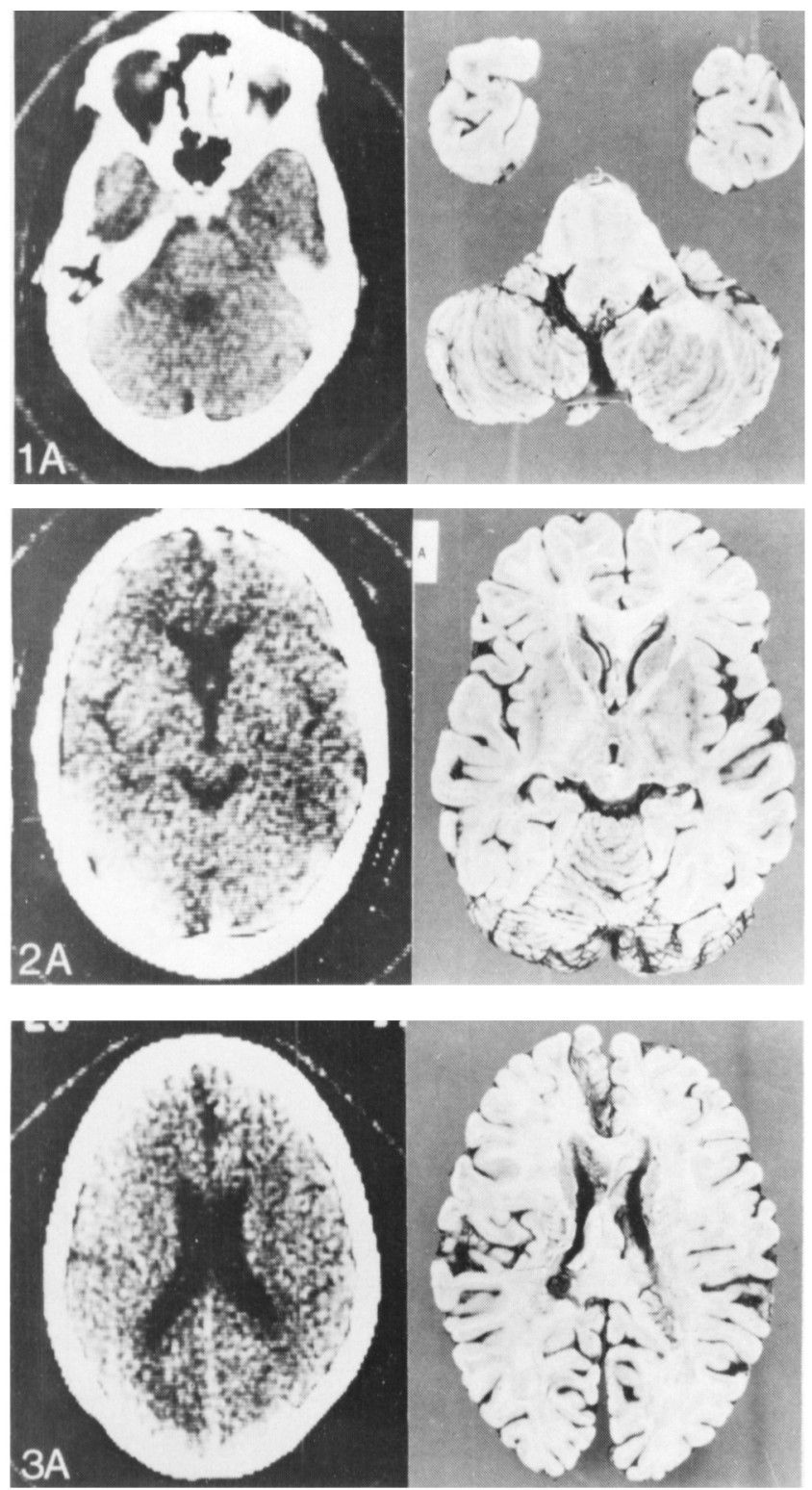
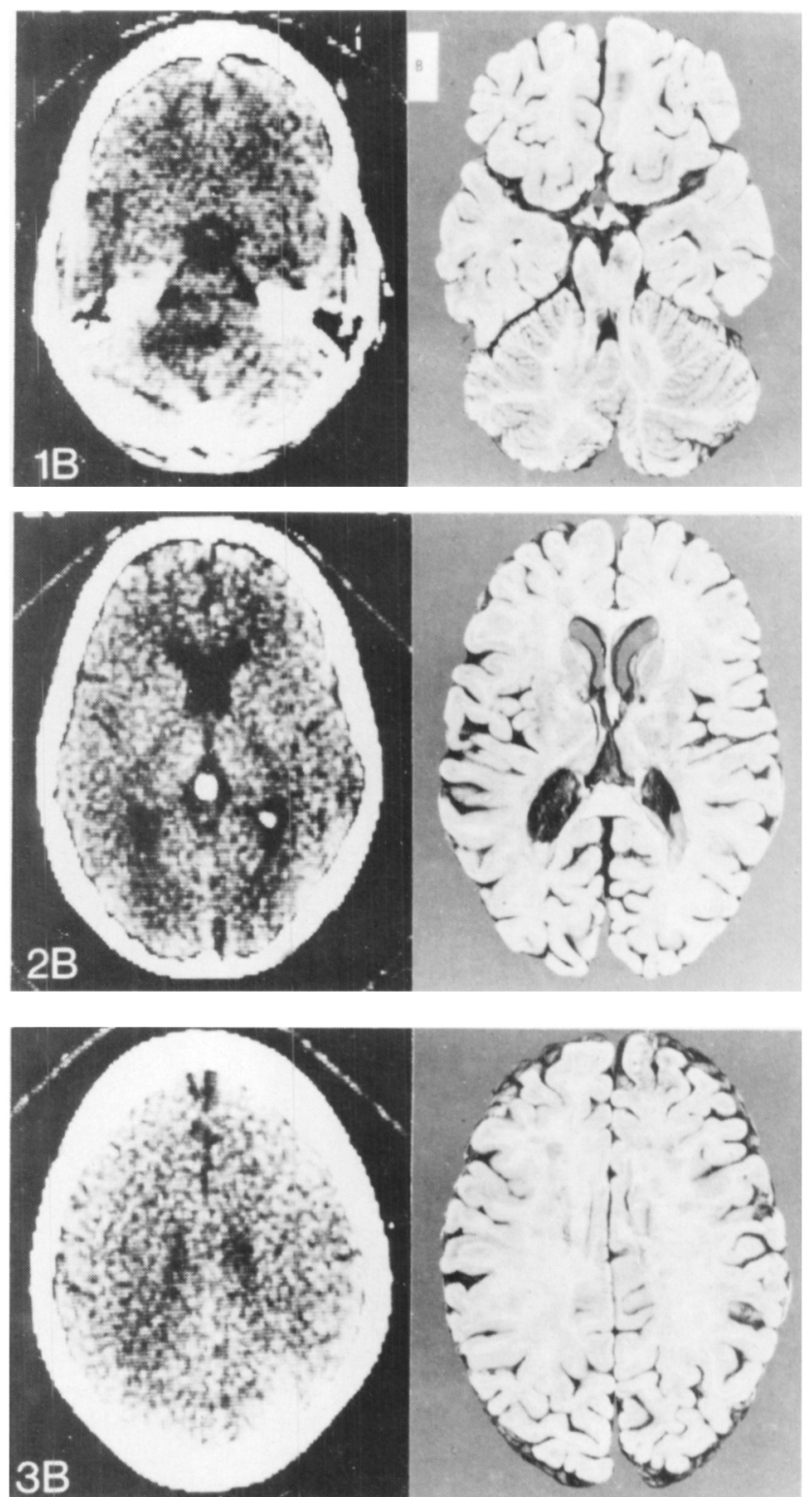

Figure l-Normal CT scan and corresponding brain sections. 

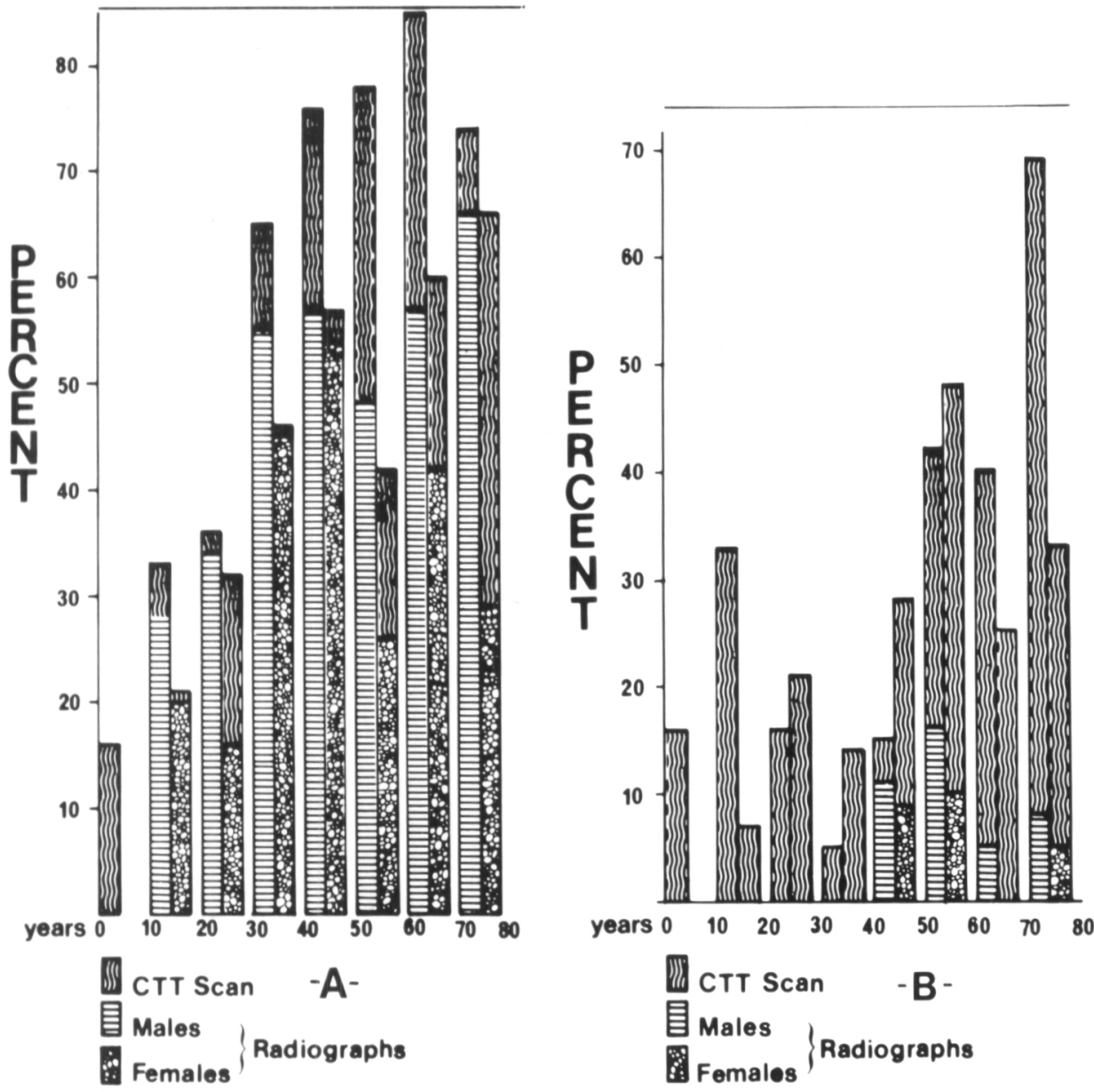

Figure 2-The incidence of pineal (A) and choroid plexus (B) identification on the CT scan and conventional radiographs based on the analysis of 500 normal cases.

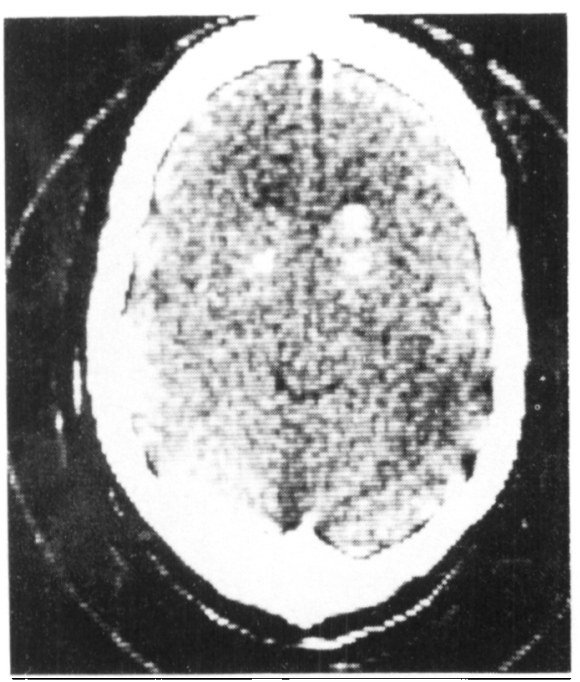

Figure 3-Bilateral asymptomatic calcification in the head of caudate nucleus in a 30 year old female patient with otherwise normal skull radiographs.
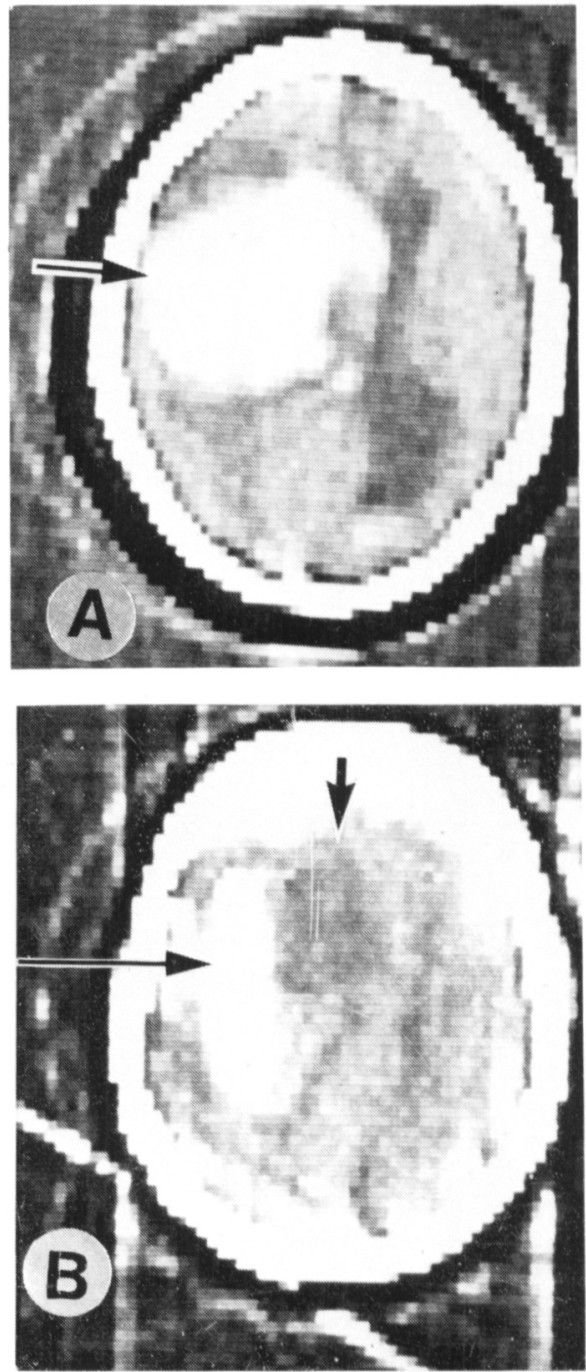

Figure 4-Intracerebral hemorrhage. This appears as a homogeneous high density area which may be globular or elliptical in shape (horizontal arrows). An adjacent area of brain edema may be seen (vertical arrow).
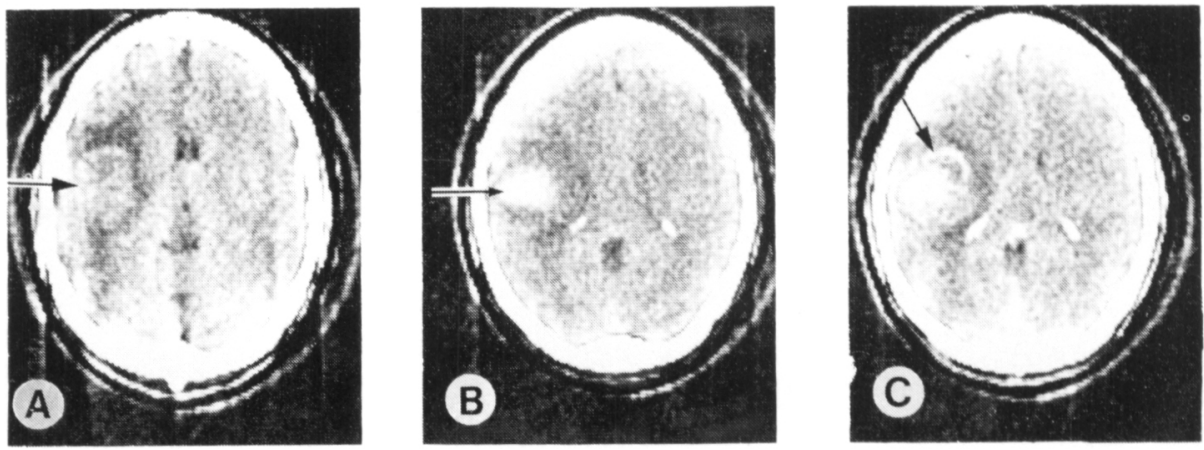

Figure 5-Atypical appearances of intracerebral hemorrhage. (A) shows a deep temporal lesion with a high density area in its center shown at a higher section (B). This is surrounded by brain edema which is partially contrast enhanced (arrow in $\mathrm{C}$ ). At operation an intracerebral haematoma was evacuated with almost complete recovery. 

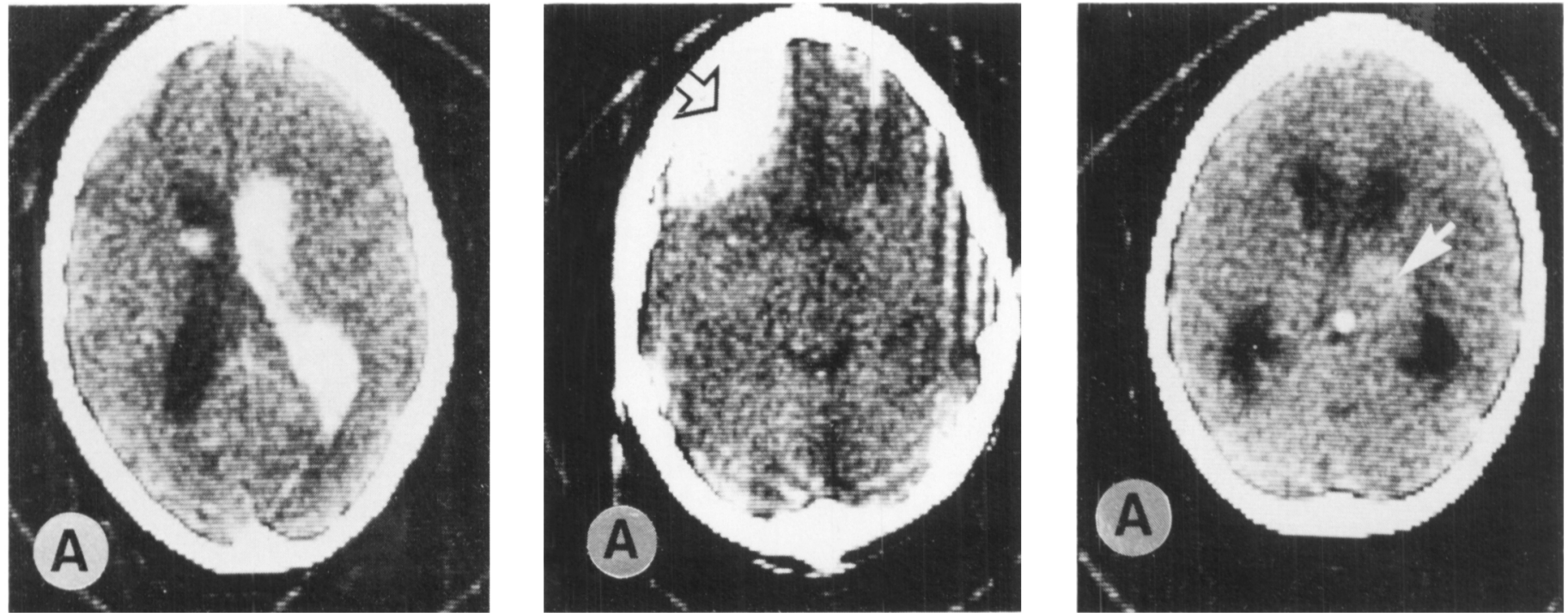

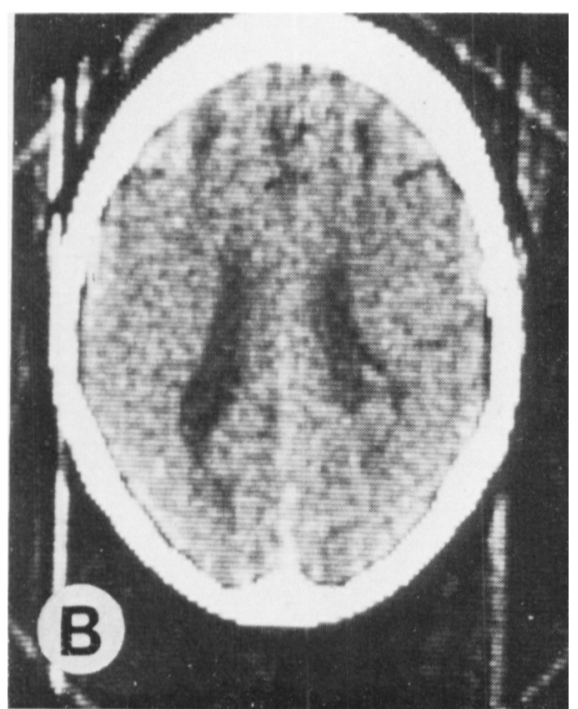

Figure 6-Intraventricular hemorrhage. (A) shows a primary hematoma within the right lateral ventricle. It was extending into the third and fourth ventricles on lower sections (not shown). Repeat scan three weeks later shows complete absorption of the hematoma. The patient is a 53 year old man in whom no bleeding source could be detected. At present he has only a mild neurological deficit.

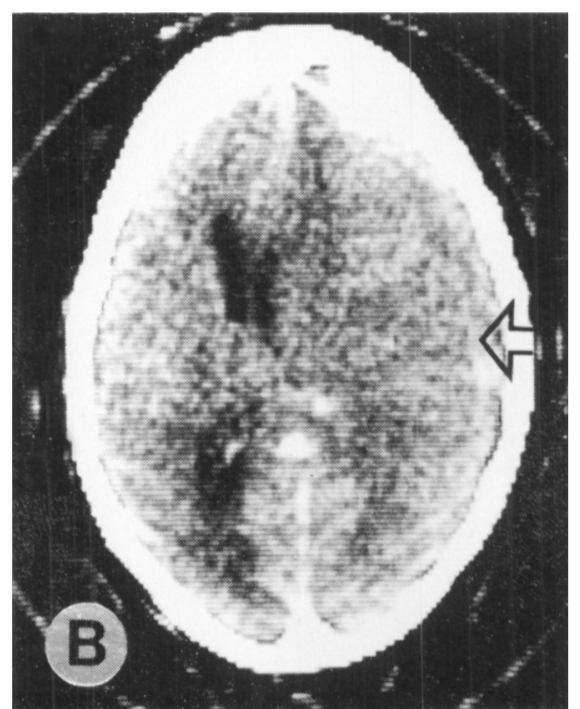

Figure 7-Extracerebral hemorrhage. (A) extradural and (B) relatively subacute subdural hematomas. Note that as the lesion becomes less acute its density approaches that of the brain.

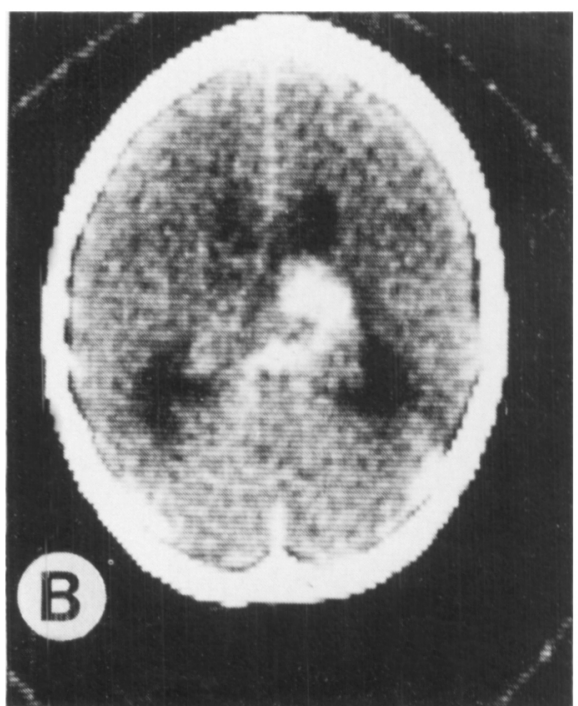

Figure 8-Arterio-venous malformation. (A) before and (B) after contrast enhancement. The nature of the lesion could only be detected at angiography. 

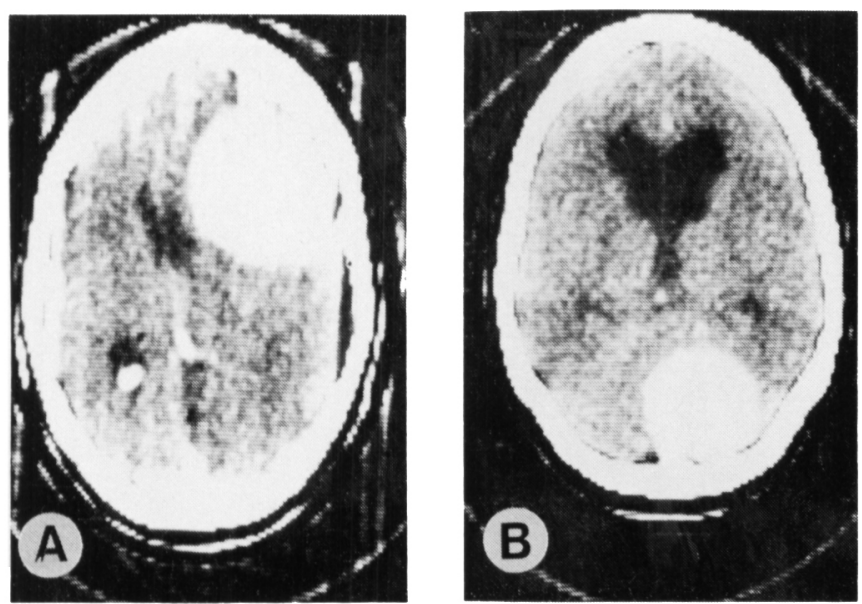

Figure 9-Typical appearances of meningioma. (A) right frontal and $(B)$ posterior fossa meningioma. Both lesions are contrast enhanced.
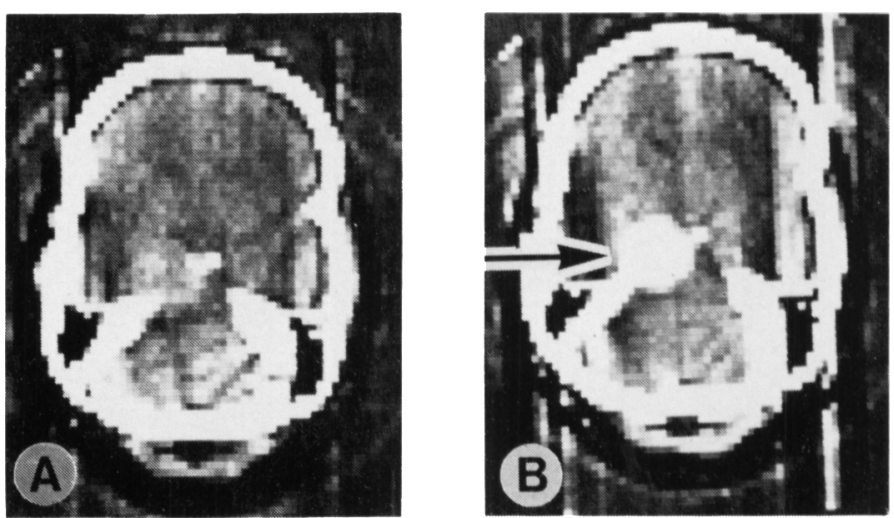

Figure 10-Tentorial meningioma. This example illustrates the importance of contrast enhancement. The tumor shown in (B) can hardly be identified prior to contrast enhancement in $(A)$.
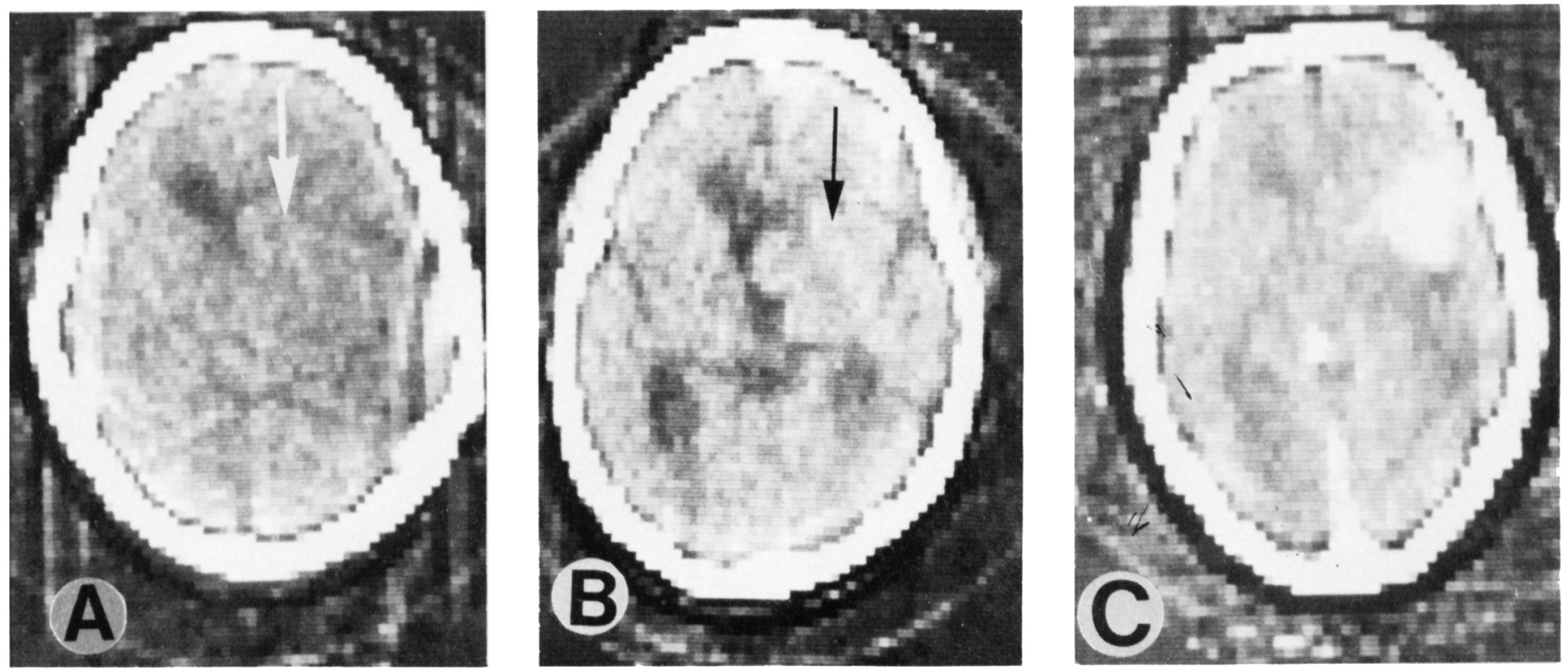

Figure /I-Meningioma simulating glioma on the CT scan. (A) and (B) depict two roughly similar lesions on the preliminary scan. (A) was a subtemporal meningioma which was partially contrast enhanced (C). (B) was a deep thalamic glioma that did not show any contrast enhancement. 

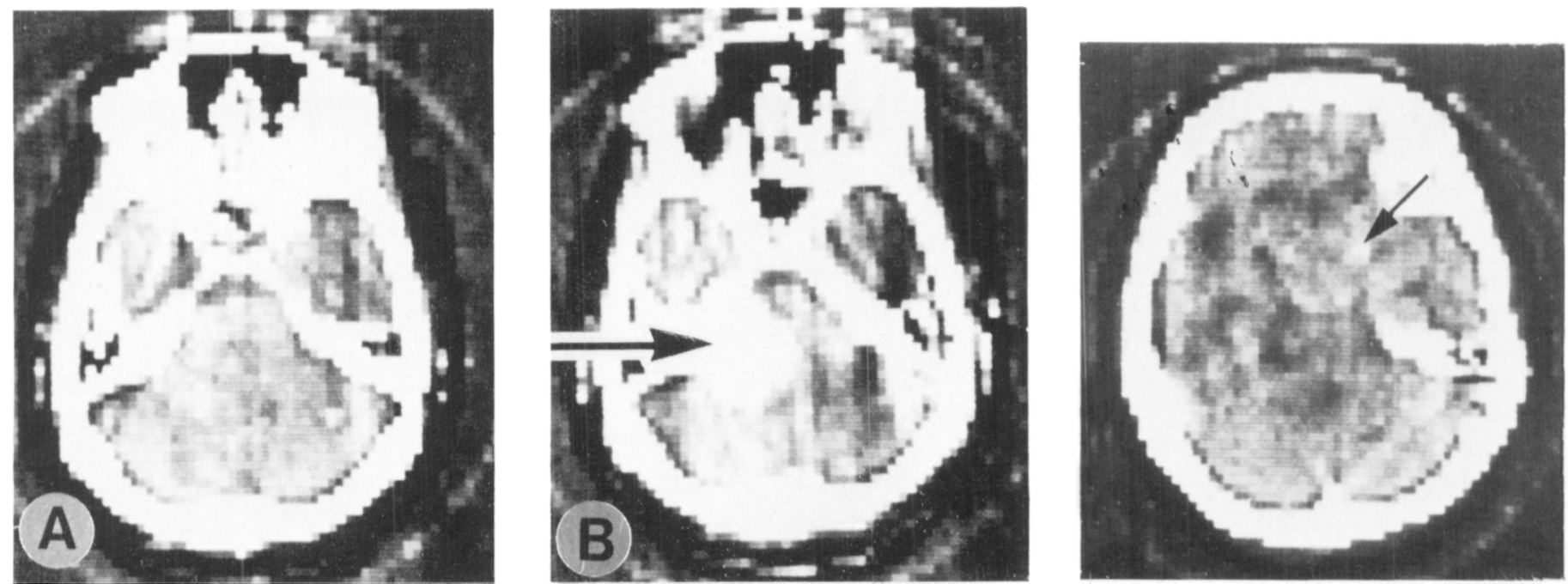

Figure 12-Acoustic neuroma. (A) before and (B) after contrast enhancement.

Figure 13-Large suprasellar pituitary adenoma (contrast enhanced).
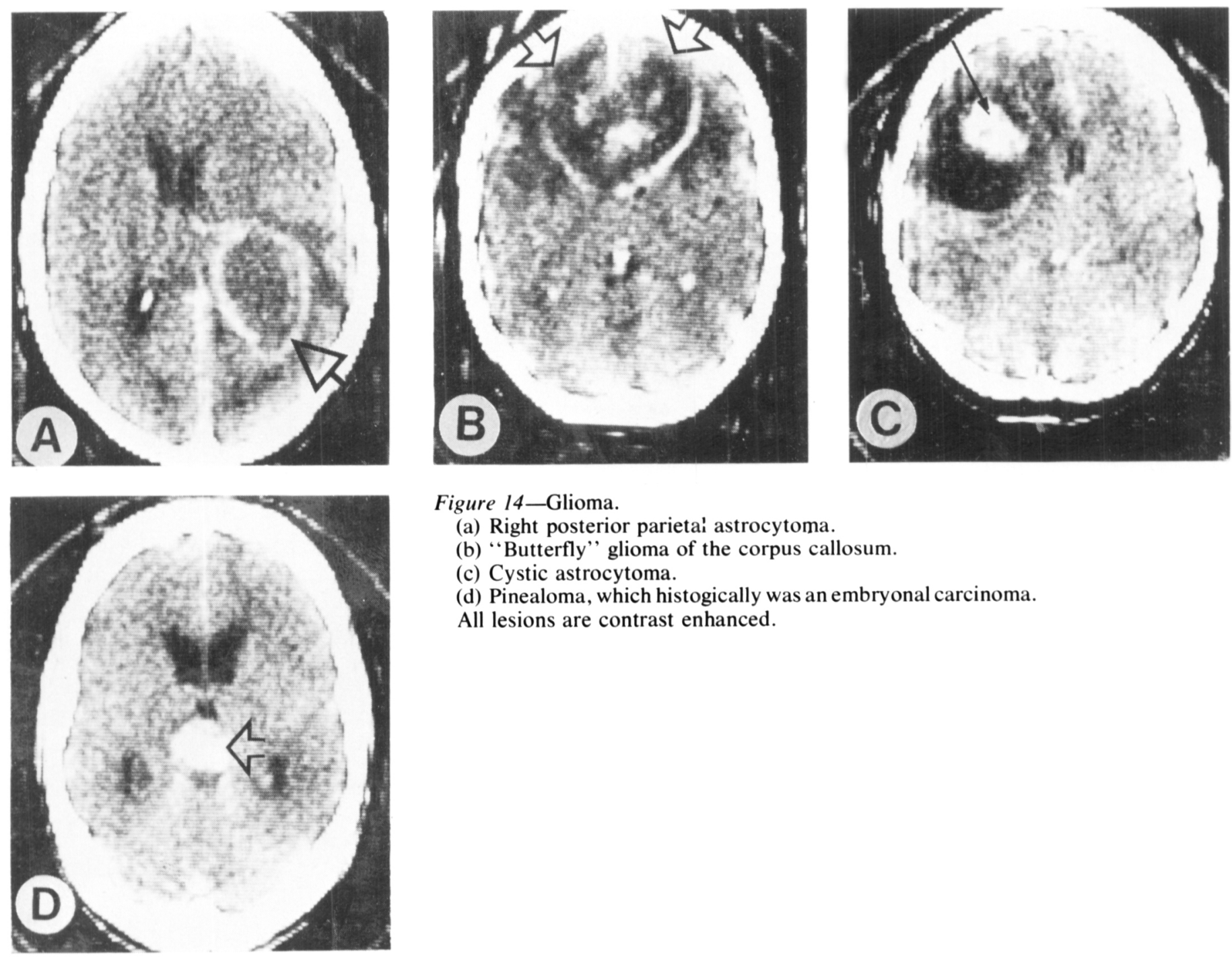

Figure 14-Glioma.

(a) Right posterior parietal astrocytoma.

(b) "Butterfly" glioma of the corpus callosum.

(c) Cystic astrocytoma.

(d) Pinealoma, which histogically was an embryonal carcinoma.

All lesions are contrast enhanced. 

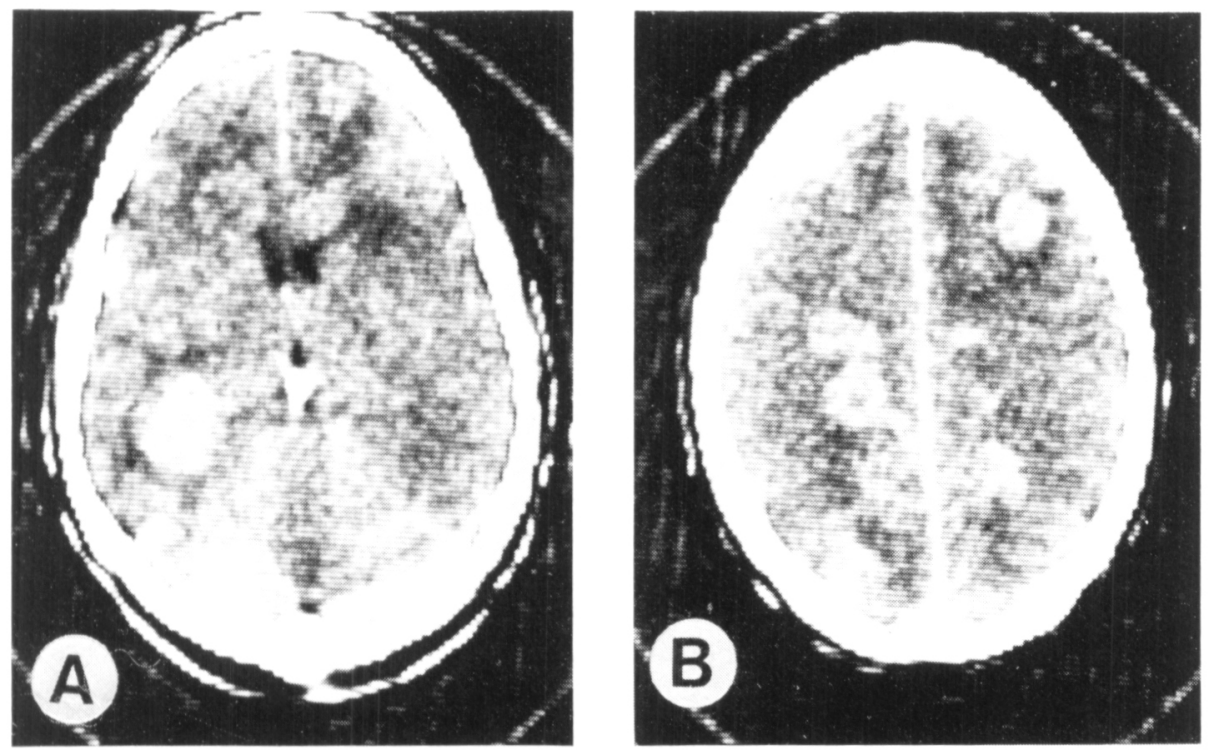

Figure 15-Cerebral metastases. A and B show numerous secondary melanoma deposits (contrast enhanced). Only one lesion could be detected with certainty on a previous angiogram.

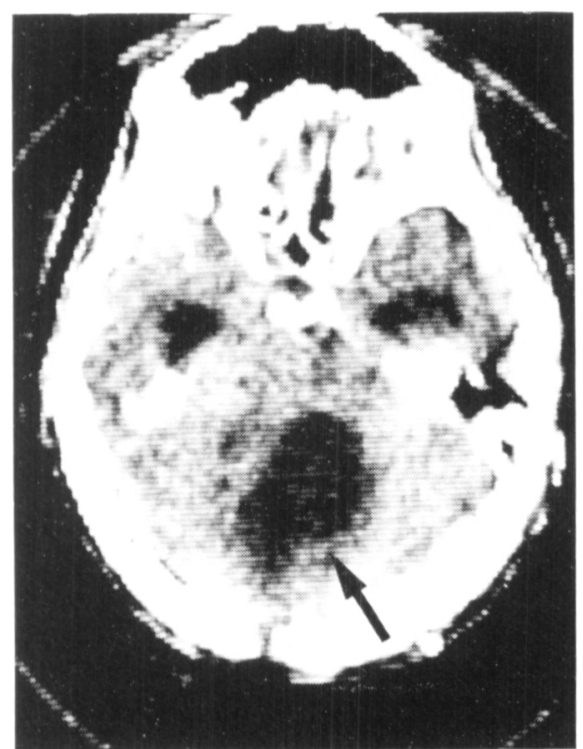

Figure 17-Posterior fossa cholesteatoma.
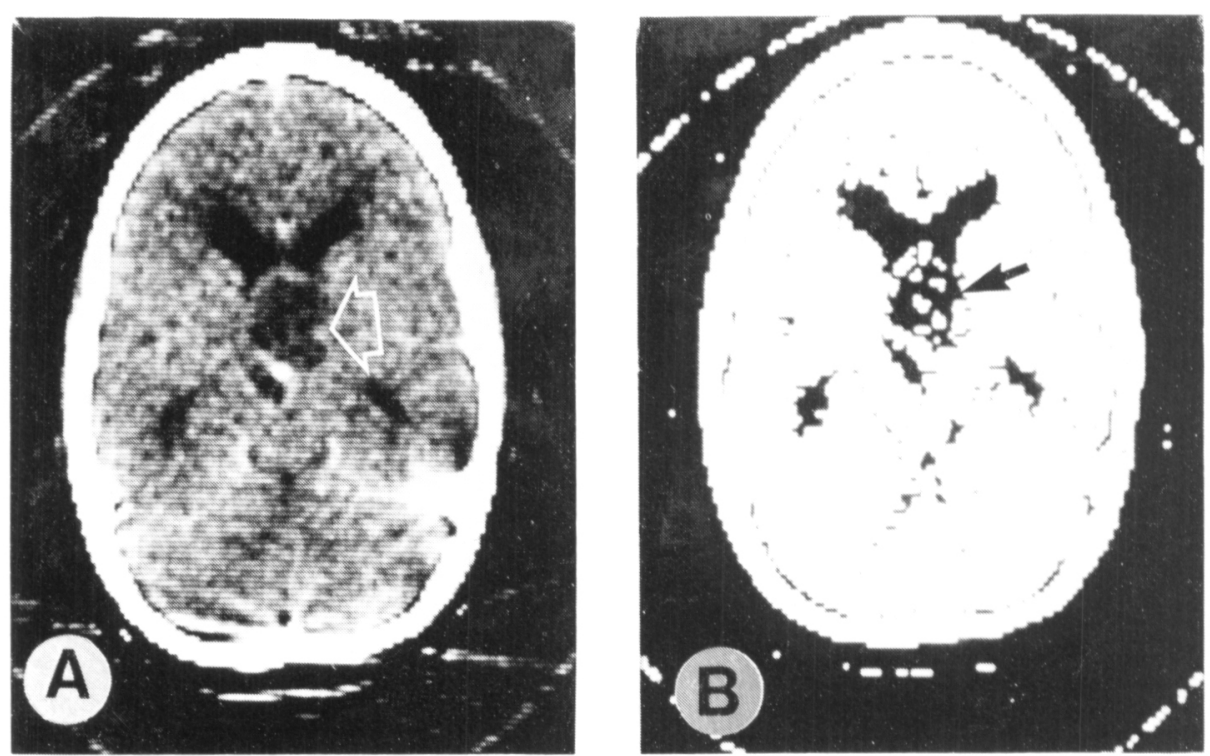

Figure 18-Cystic craniopharyngioma. Note the high density of tumor "capsule" in (A) and minute calcified foci in (B). 


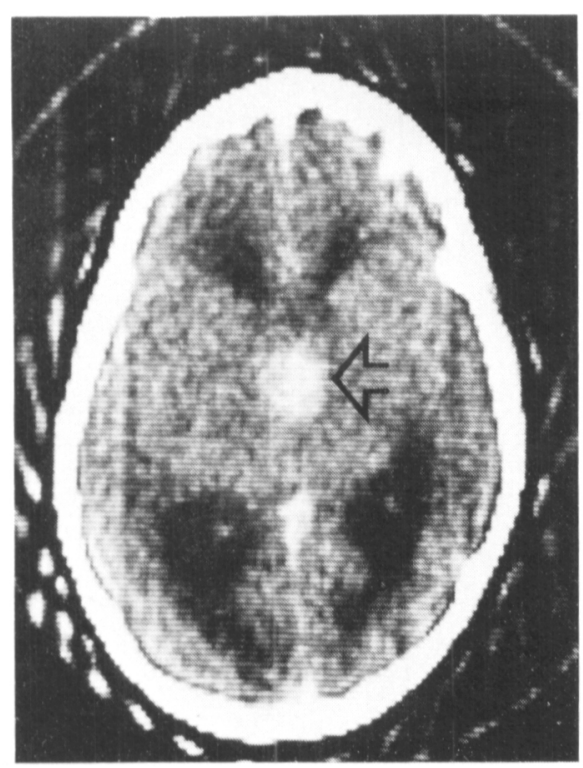

Figure 19-Optic chiasm glioma (contrast enhanced).
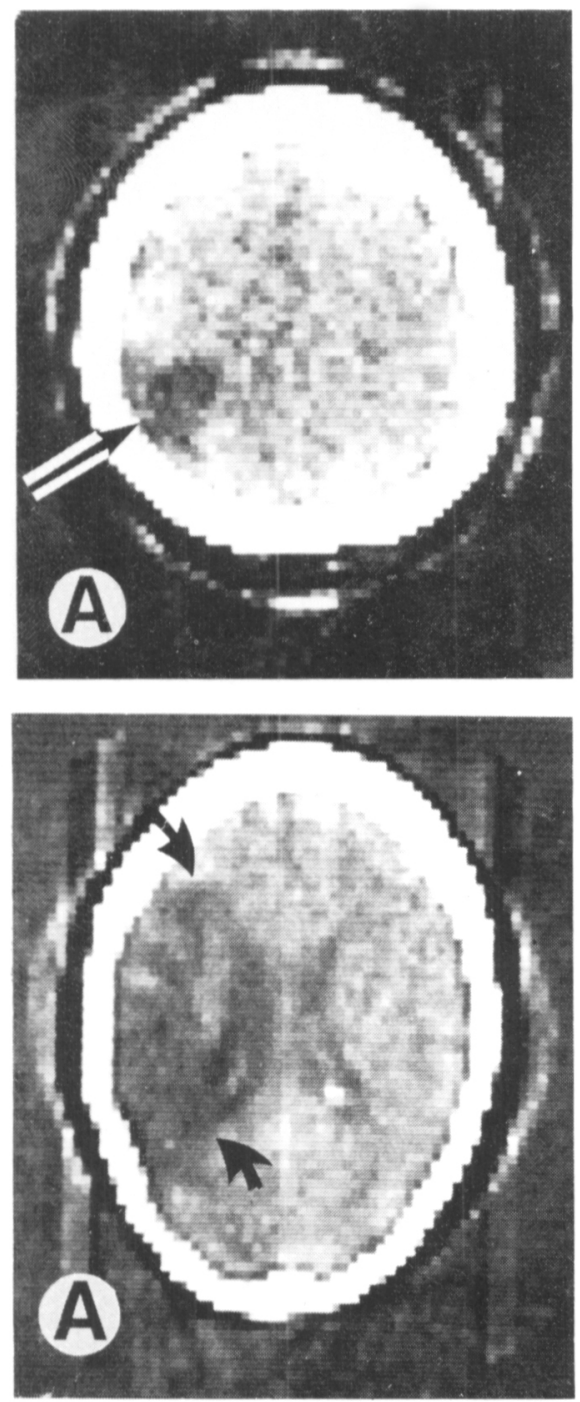
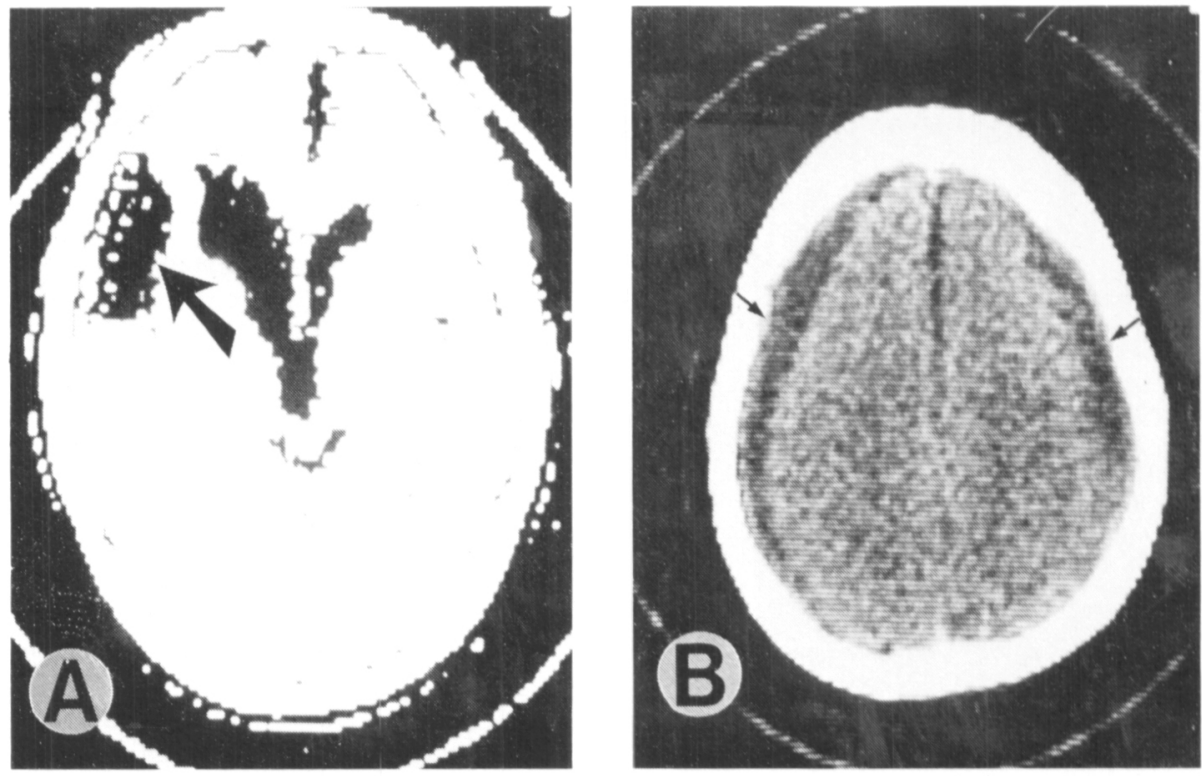

Figure 20-Chronic subdural hematoma. (A) depicts the usual biconvex shape of chronic subdural hematoma. (B) shows bilateral lesions which are semilunar in shape despite their chronicity.

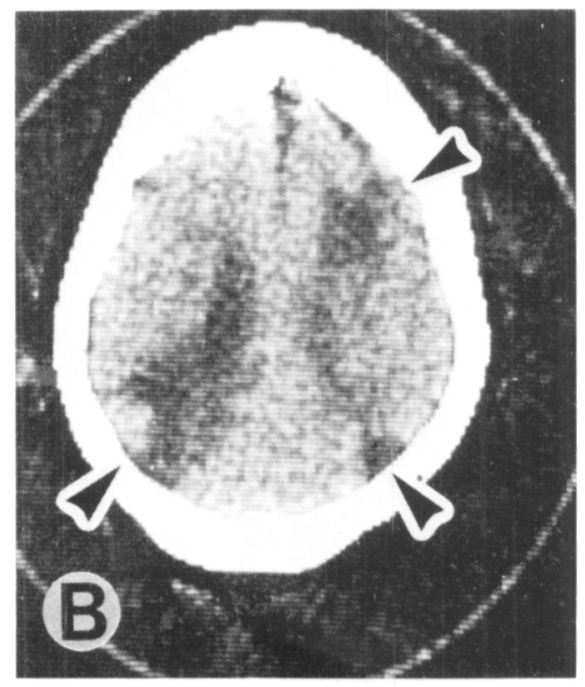

Figure 21-Focal cerebral infarction.

(a) single wedge-shaped lesion.

(b) multiple lesions.

Figure 22-Differentiation between infarction and infiltrating glioma.

(a) Massive cerebral infarction.

(b) Extensive infiltrating oligodendroglioma.

Although the two lesions are roughly similar in shape, sparing of the brain cortex is not a feature of infarction but it may be seen in malignancy .

(c) Chronic cerebral infarction. Note ipsilateral ventricular dilatation and displacement of midline structures towards the side of the lesions.

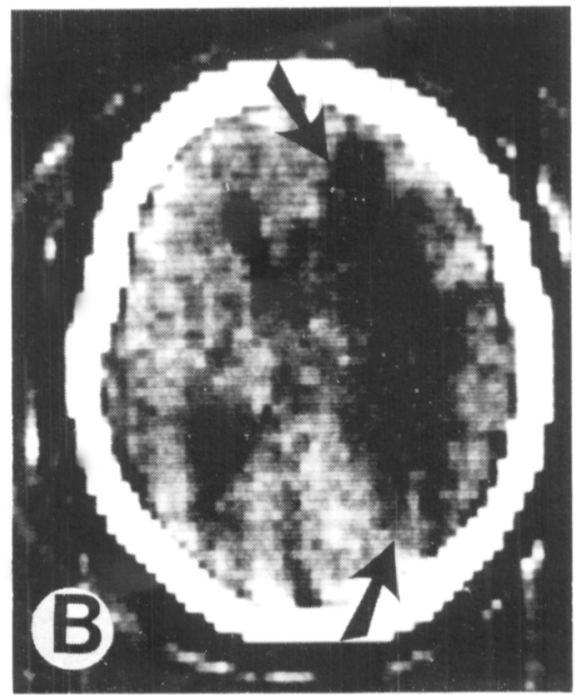



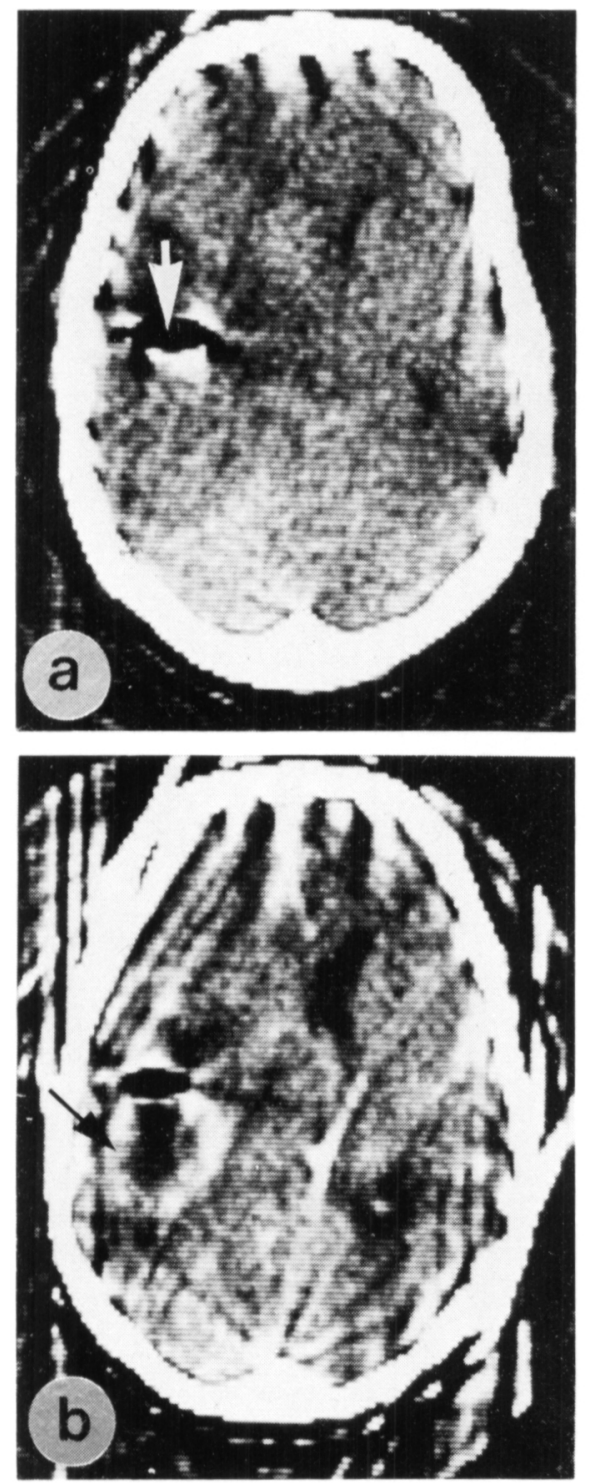

Figure 23-Brain abscess. The lesion developed about one month following mastoidectomy. (A) before and (B) after contrast enhancement. White arrow points to some air within the abscess cavity. Black arrow points to the abscess wall.

\section{REFERENCES}

BAKER, H. L. Jr., CAMPBELL, J. K., HOUSER, D. W., REESE, D. F., SHEEDY, P. F., HOLMAN, C. B., with the technical assistance of KURLAND, $R$. L. (1974). Computer assisted tomography of the head. An early evaluation. Mayo Clinic Proceedings: 49, 17-27.

BANNA, M. and PORTEOUS, C. (1976). Basic introduction to computerized tomography. Accepted for publication. The Canadian Journal of Radiology.

DAVIS, D. O. and PRESSMAN, B. D. (1974). Computerized tomography of the
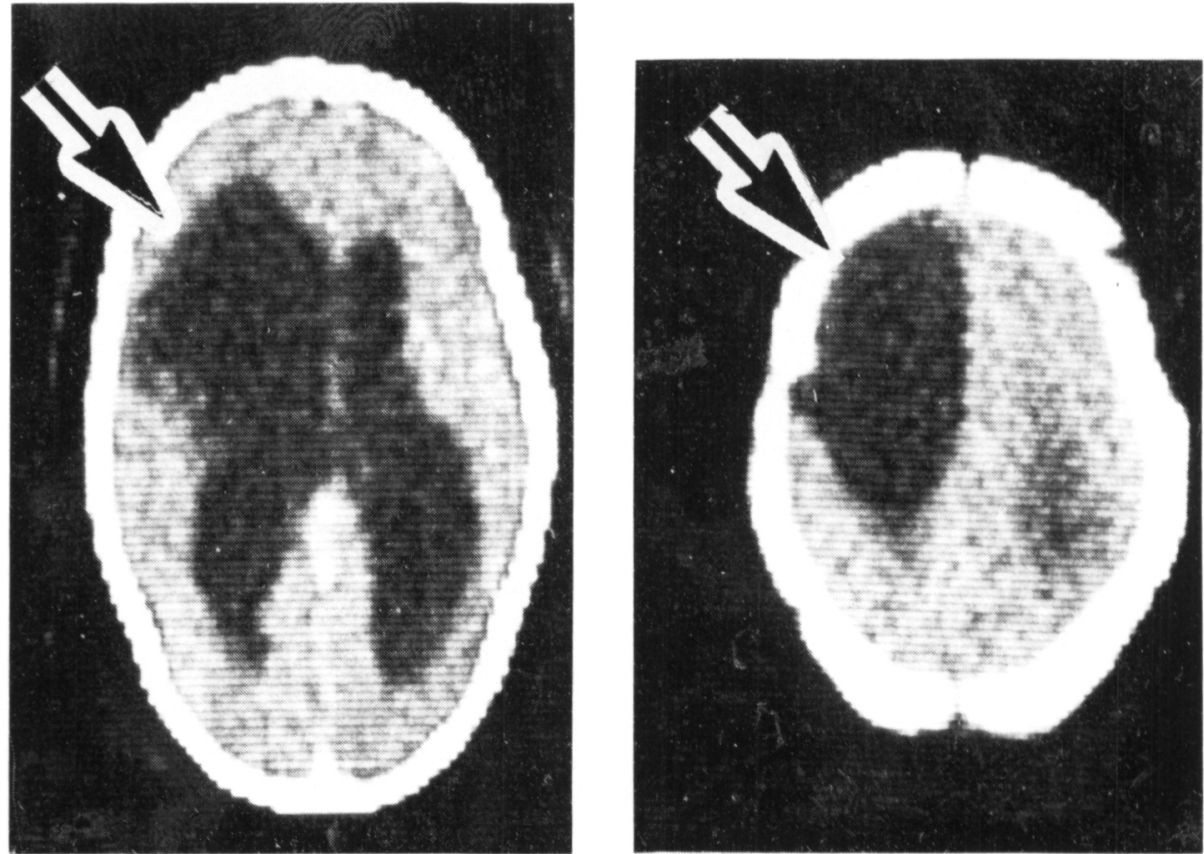

Figure 24-Post-traumatic hydrocephalus in a 5 month old baby following difficult delivery. Arrow points to a porencephalic cyst.
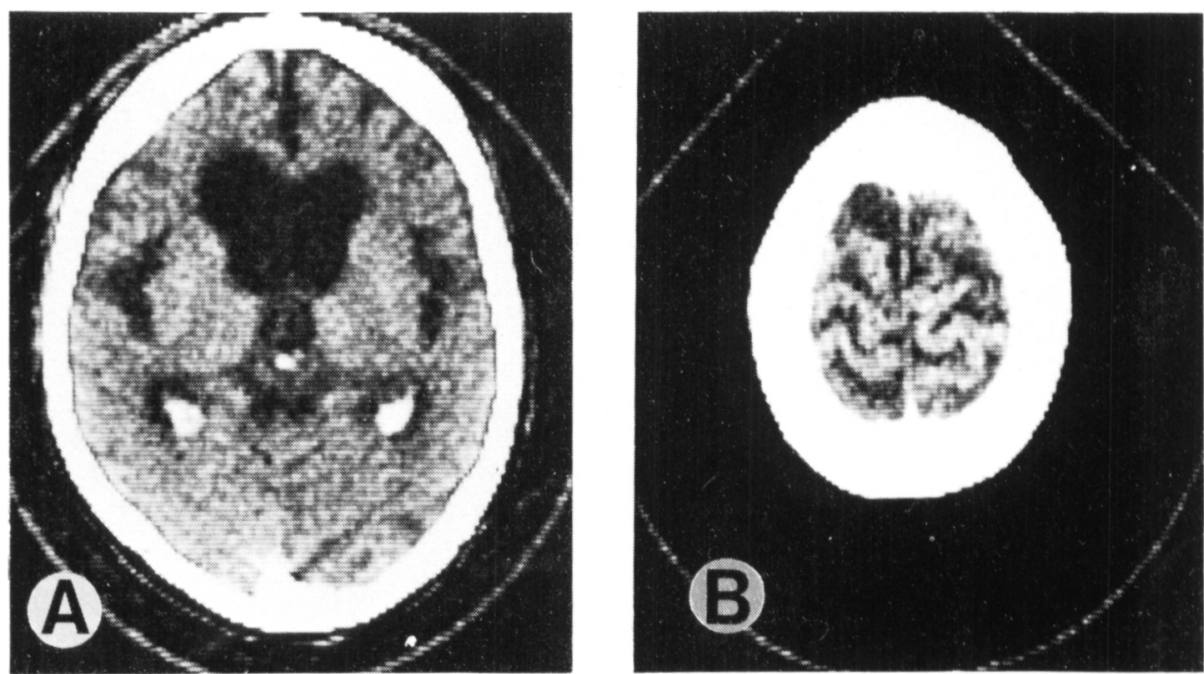

Figure 25-Advanced brain atrophy. (A) shows generalized ventricular dilatation and wide Sylvian fissues. (B) is the upper most scan showing widening of the cerebral sulci.

brain. Radiologic Clinic of North America: 12, 297-313

PAXTON, R. and AMBROSE, J. (1974). The EMI scanner. A brief review of the first 650 patients. British Journal of Radiology: 47, 530-565.

SAGE., M. R., McALLISTER, V. L., KENDALL, B. E., BULL, J. W. D. and MOSELEY, I. F. (1975). Radiology in the diagnosis of colloid cysts of the third ventricle. British Journal of Radiology: 48, 708-723. 